\title{
Synthesis, characterization and in vitro antibacterial activity of novel phthalazine sulfonamide derivatives
}

\author{
MARZIEH ABBASI ${ }^{\mathrm{a}, \mathrm{d}}$, SEYED MOHAMAD REZA NAZIFI ${ }^{\mathrm{b}},{\text { ZAHRA SADAT NAZIFI }{ }^{\mathrm{a}, \mathrm{c}, *} \text { and }}$ \\ AHMAD REZA MASSAH ${ }^{\mathrm{a}, \mathrm{c}, *}$ \\ ${ }^{a}$ Department of Chemistry, Islamic Azad University, Shahreza Branch, Shahreza, Isfahan 86145-311, \\ Islamic Republic of Iran \\ ${ }^{b}$ Department of Medicinal Chemistry, School of Pharmacy and Pharmaceutical Sciences, Isfahan University of \\ Medical Sciences, Isfahan, Islamic Republic of Iran \\ ${ }^{\mathrm{c}}$ Razi Chemistry Research Center, Islamic Azad University, Shahreza Branch, Shahreza, Isfahan 86145-311, \\ Islamic Republic of Iran \\ ${ }^{\mathrm{d} Y o u n g}$ Researchers and Elite Club, Islamic Azad University, Shahreza Branch, Shahreza, Isfahan, \\ Islamic Republic of Iran \\ E-mail: massah@iaush.ac.ir; nazifi.zahrasadat@gmail.com
}

MS received 7 January 2017; revised 23 May 2017; accepted 31 May 2017

\begin{abstract}
Several phthalazine derivatives were synthesized by the one-pot three-component condensation in good to high yields in the presence of diatomite- $\mathrm{SO}_{3} \mathrm{H}$ as a solid acid catalyst. Then, a series $(\mathrm{n}=14)$ of phthalazine sulfonamides were synthesized by the reaction phthalazine sulfonyl chloride and various amines under solvent-free conditions. The prepared compounds were screened for antibacterial activity against Escherichia coli (E. coli ATCC 25922) and Staphylococcus aureus (S. aureus ATCC 5213) as gram negative and positive respectively. Also, in silico physicochemical parameters of synthesized compounds were studied to predict absorption and permeability using Molinspiration online property calculation server.
\end{abstract}

Keywords. Phthalazinesulfonamide; aldehyde; dimedone; sulfonated diatomite; heterogeneous catalyst.

\section{Introduction}

Sulfonamides have received considerable attention in recent years due to their wide range of biological properties. Sulfonamides derivatives are known to exhibit various pharmacological activities such as anticonvulsants ${ }^{1}$ IV protease inhibitors, ${ }^{2}$ anticancer, ${ }^{3}$ antibacterial, ${ }^{4}$ antiinflammatory ${ }^{5}$ and antitumor agents. ${ }^{6}$ Pharmaceutically important examples include the protease inhibitor amprenavir, analgesic celecoxib, sildenafil for erectile dysfunction and antimigraine agent sumatriptan. ${ }^{7}$

Although many efforts have been made towards the development of novel sulfonamides, the conventional synthesis involves the reaction of amino compounds with sulfonyl chlorides. ${ }^{8}$

In the past few decades, heterocyclic chemistry has been one of the most important disciplines in organic synthesis and pharmaceutical chemistry. ${ }^{9}$ Among a large variety of heterocyclic compounds, heterocycles containing phthalazine moiety has many applications in medicinal chemistry ${ }^{10}$ (Figure 1).

Furthermore, phthalazine derivatives show pharmacological activities such as antimicrobial, ${ }^{11}$ anticonvulsant, ${ }^{12}$ antifungal, ${ }^{13}$ anticancer, ${ }^{12}$ anti-tumor ${ }^{14}$ and anti-inflammatory activities. ${ }^{15}$ A number of catalysts have been reported for the synthesis of phthalazine such as: silica sulfuric acid, ${ }^{16} \mathrm{~N}$-halo sulfonamide, ${ }^{17}$ microwave, ${ }^{18}$ ultrasonic in 1 -butyl-3-methylimidazolium bromide $((\mathrm{Bmim}) \mathrm{Br}),{ }^{19}$ nano-alumina acidic, ${ }^{20}$ ionic liquid, ${ }^{21}$ p-TSA. ${ }^{22}$ In continuation to our previous study in the synthesis of sulfonamides and heterocyclic compounds, ${ }^{23}$ a simple and efficient method for the synthesis of novel phthalazine sulfonamides $(n=14)$ by the use of sulfonated diatomite under solvent-free conditions has been described (Scheme 1). Also, the in vitro antibacterial activity of the synthesized compounds is evaluated against Gram-negative (E. coli) and Gram-positive $(S$. aureus) bacteria.

\footnotetext{
*For correspondence
}

Electronic supplementary material: The online version of this article (doi:10.1007/s12039-017-1337-8) contains supplementary material, which is available to authorized users. 


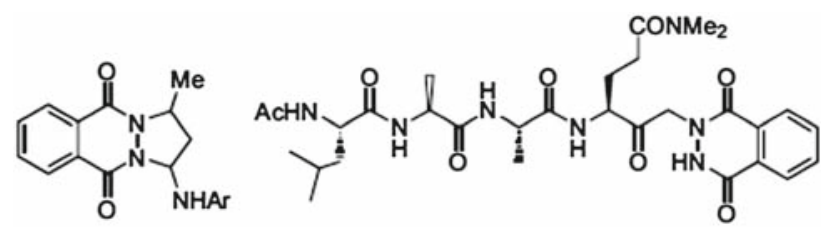

Figure 1. Antihypoxic and antipyretic agent, HAV3C inhibitor.

\section{Experimental}

\subsection{General}

All chemicals were purchased from Merck chemical company in high purity. IR spectra were obtained as $\mathrm{KBr}$ pellets on a Perkin-Elmer FT-IR spectrophotometer. ${ }^{1} \mathrm{H}$ NMR and ${ }^{13} \mathrm{C}$ NMR were recorded either $\mathrm{CDCl}_{3}$ or DMSO- $\mathrm{d}_{6}$ solvents on Bruker with tetramethylsilane (TMS) as an internal standard. Melting points were taken in open capillary tubes. The purity determination of the products and reaction monitoring were accomplished by TLC on silica gel polygram (from Merck Company). The phthalhydrazid was synthesized according to the reported method. ${ }^{24}$

\subsection{Preparation of diatomite $-\mathrm{SO}_{3} \mathrm{H}$}

Diatomite- $\mathrm{SO}_{3} \mathrm{H}$ was prepared according to the previously reported method. ${ }^{25}$ In a typical experiment, diatomite was dried and activated in vacuum at $100^{\circ} \mathrm{C}$. Activated diatomite $(2 \mathrm{~g})$ was dispersed in dry $\mathrm{CH}_{2} \mathrm{Cl}_{2}$ and chlorosulfonic acid $(1 \mathrm{~mL})$ was added to the solution at room temperature. After $2 \mathrm{~h}$, the white solid was filtered, washed repeatedly by dry $\mathrm{CH}_{2} \mathrm{Cl}_{2}$, and dried at $120^{\circ} \mathrm{C}$ for $12 \mathrm{~h}$.

\subsection{General procedure for the synthesis of $2 H$-indazolo $(2,1-b)$ phthalazine-triones}

A mixture of dimedone $(0.14 \mathrm{~g}, 1 \mathrm{mmol})$, phthalhydrazide $(0.16 \mathrm{~g}, 1 \mathrm{mmol})$, aromatic aldehyde $(1 \mathrm{mmol})$ and diatomite$\mathrm{SO}_{3} \mathrm{H}(0.3 \mathrm{~g})$ in ethanol was heated at $80^{\circ} \mathrm{C}$. The progress of the reaction was monitored by TLC ethyl acetate:n-hexane (1:1). After completion of the reaction, the diatomite- $\mathrm{SO}_{3} \mathrm{H}$ was filtered from the reaction mixture. After evaporation of the solvent, the crude product was purified by recrystallization in aqueous ethanol to afford the pure product.

\subsection{General procedure for the synthesis of phthalazine sulfonyl chloride $(5 a, 5 b)$}

$\mathrm{ClSO}_{3} \mathrm{H}(15 \mathrm{mmol})$ was added to phthalazine $(1 \mathrm{mmol})$ and stirred at room temperature for a minute. The progress of the reaction was monitored by TLC. After completion of the reaction, ice was added to the reaction mixture, and the crystalline product was separated by filtration and was used in the next step without any purification.

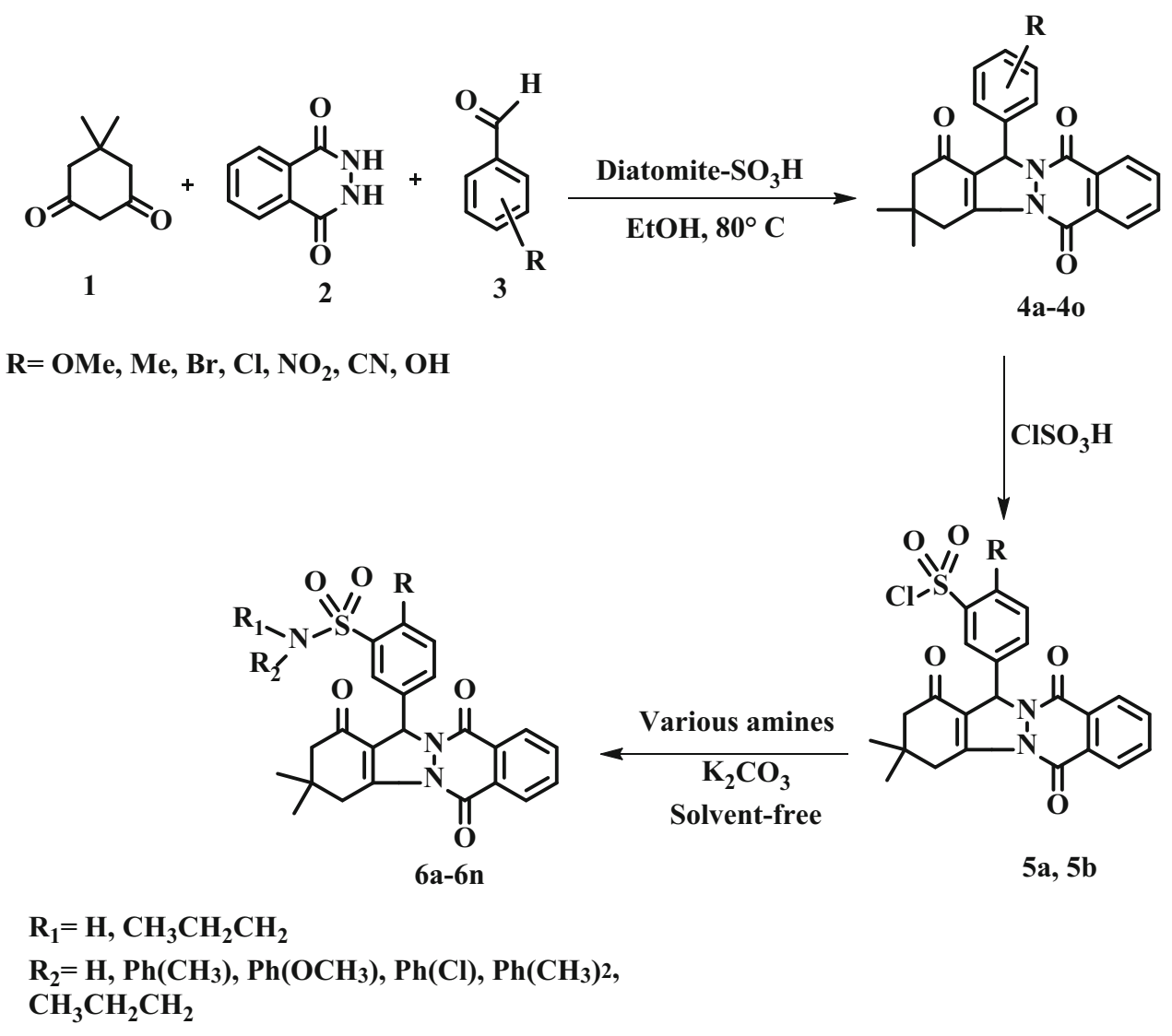

Scheme 1. Preparation of phthalazine sulfonamides. 
5-(2,2-dimethyl-4,7,12-trioxo-1,2,3,4,5,7,12,14-octahydrophthalazino(2,3-b)phthalazin-5-yl)-2-methoxybenzene-1sulfonyl chloride (5a): M.p.: 233-235 ${ }^{\circ}$; IR $\left(\mathrm{cm}^{-1}\right)$ : $v_{\mathrm{C}-\mathrm{H}} 2960, v_{\mathrm{C}=\mathrm{O}} 1666, v_{\mathrm{C}=\mathrm{O}} 1630, v_{\mathrm{C}=\mathrm{C}} 1605, v_{\mathrm{SO} 2} 1363$, $1172 ;{ }^{1} \mathrm{H} \mathrm{NMR}(\delta, \mathrm{ppm}$ in DMSO-d 6$): 1.1(\mathrm{~s}, 6 \mathrm{H}), 2.2(\mathrm{~s}, 2 \mathrm{H})$, $3.1(\mathrm{~d}, 1 \mathrm{H}, J=20 \mathrm{~Hz}), 3.3(\mathrm{~d}, 1 \mathrm{H}, J=20 \mathrm{~Hz}), 3.7(\mathrm{~S}, 3 \mathrm{H})$, $6.2(\mathrm{~s}, 1 \mathrm{H}), 6.9(\mathrm{~d}, 1 \mathrm{H}, J=8 \mathrm{~Hz}), 7.4\left(\mathrm{dd}, 1 \mathrm{H}, J_{1}=12 \mathrm{~Hz}\right.$, $\left.J_{2}=2.4 \mathrm{~Hz}\right), 7.6(\mathrm{~d}, 1 \mathrm{H}, J=2 \mathrm{~Hz}), 7.9(\mathrm{~m}, 1 \mathrm{H}), 8 .(\mathrm{m}, 1 \mathrm{H})$, $8.2(\mathrm{~m}, 1 \mathrm{H}) ;{ }^{13} \mathrm{C}$ NMR $(\delta, \mathrm{ppm}$ in DMSO-d 6$): 27.7,28.1$, 34.2, 37.1, 50.2, 55.4, 63.8, 111.6, 117.4, 126.7, 127.3, 127.5, $127.9,128.6,128.8,129.9,133.7,134.3,134.6,151 ., 153.6$, 155.3, 156., 191.9.

5-(2,2-dimethyl-4,7,12-trioxo-1,2,3,4,5,7,12,14-octahydrophthalazino(2,3-b)phthalazin-5-yl) -2-methylbenzene-1-sulfonyl chloride (5b): M.p.: $235-237^{\circ} \mathrm{C}$; IR $\left(\mathrm{cm}^{-1}\right)$ : C-H 2959, $v \mathrm{C}=\mathrm{O} \quad 1666, v_{\mathrm{C}=\mathrm{O}} 1635, v_{\mathrm{C}=\mathrm{C}} 1606, v_{\mathrm{SO} 2} 1363$, $1173 ;{ }^{1} \mathrm{H}$ NMR $(\delta$, ppm in DMSO-d 6$): 1.1(\mathrm{~s}, 3 \mathrm{H}), 1.1(\mathrm{~s}, 3 \mathrm{H})$, 2.2(s, 2H), $2.4(\mathrm{~s}, 3 \mathrm{H}), 3.1\left(\mathrm{dd}, 1 \mathrm{H}, \underline{J}_{1}=20 \mathrm{~Hz}, J_{2}=1 . \mathrm{Hz}\right)$, $3.3(\mathrm{~d}, 1 \mathrm{H}, J=20 \mathrm{~Hz}), 6.2(\mathrm{~s}, 1 \mathrm{H}), 7(\mathrm{~d}, 1 \mathrm{H}, J=4 \mathrm{~Hz}), 7.2$ $\left(\mathrm{dd}, 1 \mathrm{H}, J_{1}=12 \mathrm{~Hz}, J_{2}=2 \mathrm{~Hz}\right), 7.7(\mathrm{~d}, 1 \mathrm{H}, J=2 \mathrm{~Hz})$, $7.9(\mathrm{~m}, 2 \mathrm{H}), 8(\mathrm{~m}, 1 \mathrm{H}), 8.2(\mathrm{~m}, 1 \mathrm{H}) ;{ }^{13} \mathrm{C} \mathrm{NMR}(\delta, \mathrm{ppm}$ in DMSO-d 6 ): 19.7, 27.7, 28, 34.2, 37.1, 50.2, 64.1, 117.4, $125.3,126.74,127.5,127.7,128.5,128.8,130.7,133.7,134$, $134.6,135.5,145.7,151.1,153.6,155.3,191.9$.

\subsection{Preparation of phthalazine sulfonamides $(6 a-6 m)$}

Amine (1mmol) and $\mathrm{K}_{2} \mathrm{CO}_{3}(3 \mathrm{mmol})$ were ground together into fine powder in a mortar. Then, phthalazine sulfonyl chloride ( $1 \mathrm{mmol}$ ) was added to the mixture under vigorous stirring at room temperature for $30 \mathrm{~min}$. The progress of the reaction was monitored by TLC (ethyl acetate:n-hexane, 1:1). After completion of the reaction, the reaction mixture was filtered, washed with water and dried.

5-(2,2-dimethyl-4,7,12-trioxo-1,2,3,4,5,7,12,14-octahydrophthalazino(2,3-b)phthalazin-5-yl)-2-methoxy-N-(p-tolyl) benzenesulfonamide (6a): M.p.: $228-230^{\circ} \mathrm{C}$; IR $\left(\mathrm{cm}^{-1}\right)$ : $v_{\mathrm{N}-\mathrm{H}} \quad 3262, v_{\mathrm{C}-\mathrm{H}} \quad 2957, v_{\mathrm{C}=\mathrm{O}} \quad 1666, v_{\mathrm{C}=\mathrm{O}} \quad 1628, v_{\mathrm{C}=\mathrm{C}}$ $1607, v_{\mathrm{SO} 2} 136,1154 ;{ }^{1} \mathrm{H}$ NMR $(\delta, \mathrm{ppm}$ in DMSO-d 6 ): 1.1 $(\mathrm{s}, 3 \mathrm{H}), 1.1$ (s, 3H), 2.1 (s, 3H), $2.2(1 \mathrm{H}, J=16 \mathrm{~Hz}), 3.1$ (dd, $\left.1 \mathrm{H}, J_{1}=24 \mathrm{~Hz}, J_{2}=2 \mathrm{~Hz}\right), 3.3(\mathrm{~d}, 1 \mathrm{H}), 3.8(\mathrm{~S}, 3 \mathrm{H}), 6.3$ (s, 1H), 6.9(m, 4H), $7.1(\mathrm{~d}, 1 \mathrm{H}, J=8 \mathrm{~Hz}), 7.6(\mathrm{dd}, 1 \mathrm{H}$, $\left.J_{1}=8 \mathrm{~Hz}, J_{2}=2.4 \mathrm{~Hz}\right), 7.8(\mathrm{~d}, 1 \mathrm{H}, J=2.4 \mathrm{~Hz}), 8(\mathrm{~m}$, $2 \mathrm{H}), 8.1(\mathrm{~m}, 1 \mathrm{H}), 8.2(\mathrm{~m}, 1 \mathrm{H}), 9.9(\mathrm{~s}, 1 \mathrm{H}) ;{ }^{13} \mathrm{C} \mathrm{NMR}(\delta$, ppm in DMSO-d 6 ): 20.7, 28.1, 28.6, 34.7, 37.7, 50.6, 56.7, 63.8, 113.2, 117.1, 119.3, 126.8, 127.2, 128.06, 129.0, 129.4, $129.5,129.7,129.8,132.6,134.2,134.4,135,135.7,151.97$, $154.2,155.9,156.6,192.2$.

5-(2,2-dimethyl-4,7,12-trioxo-1,2,3,4,5,7,12,14-octahydrophthalazino(2,3-b)phthalazin-5-yl)-2-methoxy-N-(2-methoxyphenyl)benzenesulfonamide (6b): M.p.: $241-243^{\circ} \mathrm{C}$; IR $\left(\mathrm{cm}^{-1}\right): v_{\mathrm{N}-\mathrm{H}}$ 3301, $v_{\mathrm{C}-\mathrm{H}} 2953, v_{\mathrm{C}=\mathrm{O}} 1664, v_{\mathrm{C}=\mathrm{O}} 1631$, $v_{\mathrm{C}=\mathrm{C}} 1605, v_{\mathrm{SO} 2} 1363,1163 ;{ }^{1} \mathrm{H}$ NMR (400 MHz, DMSOd6) $\delta(\mathrm{ppm})=1(\mathrm{~s}, 3 \mathrm{H}), 1.1(\mathrm{~s}, 3 \mathrm{H}), 2.1(2 \mathrm{H}, J=16 \mathrm{~Hz})$, $3.1(\mathrm{dd}, 1 \mathrm{H}, J=2 \mathrm{~Hz}), 3.2(\mathrm{~d}, 1 \mathrm{H}), 3.5(\mathrm{~s}, 3 \mathrm{H}), 3.8(\mathrm{~s}, 3 \mathrm{H})$, $6.2(\mathrm{~s}, 1 \mathrm{H}), 6.7\left(\mathrm{dd}, 1 \mathrm{H}, J_{1}=16 \mathrm{~Hz}, J_{2}=8 \mathrm{~Hz}\right), 6.8(\mathrm{~d}$, $1 \mathrm{H}, J=8 \mathrm{~Hz}), 6.9\left(\mathrm{dd}, 1 \mathrm{H}, J_{1}=16 \mathrm{~Hz}, J_{2}=8 \mathrm{~Hz}\right), 7.1$ (m, 2H), $7.6(\mathrm{~m}, 2 \mathrm{H}), 7.9(\mathrm{~m}, 2 \mathrm{H}), 8.1(\mathrm{~m}, 1 \mathrm{H}), 8.2(\mathrm{~m}, 1 \mathrm{H})$, $8.64(\mathrm{~s}, 1 \mathrm{H}) ;{ }^{13} \mathrm{C}$ NMR $(\delta, \mathrm{ppm}$ in DMSO-d 6$): 28,28.7,34.7$, $37.7,50.6,56,56.7,63.9,111.9,113,117.2,120.7,123.1$, $126,126.23,127.2,127.6,128,129,129,129.3,129.5,134.2$, $134.4,135,151.4,151.9,154.27,155.8,156.7,192.2$.

5-(2,2-dimethyl-4,7,12-trioxo-1,2,3,4,5,7,12,14-octahydrophthalazino(2,3-b)phthalazin-5-yl)-2-methoxy-N-phenylbenzenesulfonamide (6c): M.p.: $220-222^{\circ} \mathrm{C}$; IR $\left(\mathrm{cm}^{-1}\right)$ : $v_{\mathrm{N}-\mathrm{H}} \quad 3245,\left(v_{\mathrm{C}-\mathrm{H}} \quad 2942, v_{\mathrm{C}=\mathrm{O}} 1665, v_{\mathrm{C}=\mathrm{O}} \quad 1628\right.$, $v_{\mathrm{C}=\mathrm{C}} 1604, \mathrm{vSO}_{2}$ 1364, 1154; ${ }^{1} \mathrm{H} \mathrm{NMR}(\delta, \mathrm{ppm}$ in DMSO-d 6$): 1.1(\mathrm{~s}, 3 \mathrm{H}), 1.1(\mathrm{~s}, 3 \mathrm{H}), 2.2(2 \mathrm{H}, J=16 \mathrm{~Hz}), 3.1$ $\left(\mathrm{dd}, 1 \mathrm{H}, J_{1}=20 \mathrm{~Hz}, J_{2}=2 \mathrm{~Hz}\right), 3.3(\mathrm{~d}, 1 \mathrm{H}), 3.8(\mathrm{~S}, 3 \mathrm{H})$, $6.3(\mathrm{~s}, 1 \mathrm{H}), 6.8(\mathrm{t}, 1 \mathrm{H}), 7\left(\mathrm{dd}, 2 \mathrm{H}, J_{1}=12 \mathrm{~Hz}, J_{2}=1.2 \mathrm{~Hz}\right)$, $7.1(\mathrm{~m}, 3 \mathrm{H}), 7.6\left(\mathrm{dd}, 1 \mathrm{H}, J_{1}=12 \mathrm{~Hz}, J_{2}=2.4 \mathrm{~Hz}\right), 7.8$ $(\mathrm{d}, 1 \mathrm{H}, J=2.4 \mathrm{~Hz}), 7.9(\mathrm{~m}, 2 \mathrm{H}), 8.1(\mathrm{~m}, 1 \mathrm{H}), 8.2(\mathrm{~m}, 1 \mathrm{H})$, $10.1(\mathrm{~s}, 1 \mathrm{H}) ;{ }^{13} \mathrm{C}$ NMR $(\delta, \mathrm{ppm}$ in DMSO-d 6$): 28.1,28.70$, 34.7, 37.7, 50.6, 56.7, 63.9, 113.3, 117.1, 118.9, 123.5, 126.8, $127.2,128,129,129.2,129.5,129.5,129.9,134.2,134.5,135$, 138.4, 151.9, 154.2, 155.91, 154.64, 192.2.

5-(2,2-dimethyl-4,7,12-trioxo-1,2,3,4,5,7,12,14-octahydrophthalazino(2,3-b)phthalazin-5-yl)-N-(2,4-dimethylphenyl)2-methoxybenzenesulfonamide (6d): M.p.: $185-187^{\circ} \mathrm{C}$; IR $\left(\mathrm{cm}^{-1}\right): v_{\mathrm{N}-\mathrm{H}}$ 3262, $v_{\mathrm{C}-\mathrm{H}} 2960, v_{\mathrm{C}=\mathrm{O}} 1665, v_{\mathrm{C}=\mathrm{O}} 1629$, $v_{\mathrm{C}=\mathrm{C}} 1605, v_{\mathrm{SO} 2} 1359,1158 ;{ }^{1} \mathrm{HNMR}(\delta, \mathrm{ppm}$ in DMSO-d 6 ): $1(\mathrm{~s}, 3 \mathrm{H}), 1.1(\mathrm{~s}, 3 \mathrm{H}), 2(\mathrm{~s}, 3 \mathrm{H}), 2.1(\mathrm{~s}, 3 \mathrm{H}), 2.2(2 \mathrm{H}$, $J=3.2 \mathrm{~Hz}), 3.1(\mathrm{~d}, 1 \mathrm{H}, J=2 \mathrm{~Hz}), 3.3(\mathrm{~d}, 1 \mathrm{H}, J=2.8 \mathrm{~Hz})$, $3.8(\mathrm{~s}, 3 \mathrm{H}), 6.2(\mathrm{~s}, 1 \mathrm{H}), 6.73(\mathrm{~d}, 2 \mathrm{H}, J=2 \mathrm{~Hz}), 6.9(\mathrm{~s}$, $1 \mathrm{H}), 7.1(\mathrm{~d}, 1 \mathrm{H}, J=8.8 \mathrm{~Hz}), 7.6(\mathrm{~d}, 1 \mathrm{H}, J=2.4 \mathrm{~Hz})$, $7.7\left(\mathrm{dd}, 1 \mathrm{H}, J_{1}=28 \mathrm{~Hz}, J_{2}=2.4 \mathrm{~Hz}\right), 7.9(\mathrm{~m}, 2 \mathrm{H})$, $8.1(\mathrm{~m}, 1 \mathrm{H}), 8.2(\mathrm{~m}, 1 \mathrm{H}), 9.1(\mathrm{~s}, 1 \mathrm{H}) ;{ }^{13} \mathrm{C} \mathrm{NMR}(\delta, \mathrm{ppm}$ in $\left.\mathrm{DMSO}_{-} \mathrm{d}_{6}\right): 18.0,20.8,28,28.7,34.6,37.7,50.6,56.6$, $63.9,117.2,125.9,125.9,127,127.2,128,128.5,128.6,129$, $129.4,131.5,132.9,133.03,134.00,134.07,134.24,134.3$, $135,135.4,151.8,154.2,155.8,192.1$.

N-(3-chlorophenyl)-5-(2,2-dimethyl-4,7,12-trioxo-1,2,3,4, $5,7,12,14$ octahydrophthalazino(2,3-b)phthalazin-5-yl)-2-methoxybenzenesulfonamide (6e): M.p.: $240-242^{\circ} \mathrm{C}$; IR $\left(\mathrm{cm}^{-1}\right)$ : $v_{\mathrm{N}-\mathrm{H}} \quad 3253, v_{\mathrm{C}-\mathrm{H}} \quad 2960, v_{\mathrm{C}=\mathrm{O}}$ 1665Amide, $v_{\mathrm{C}=\mathrm{O}} 1629$, $v_{\mathrm{C}=\mathrm{C}} 1599, v_{\mathrm{SO} 2} 1361,1156 ;{ }^{1} \mathrm{HNMR}(\delta, \mathrm{ppm}$ in DMSO-d 6 ): $1.1(\mathrm{~s}, 3 \mathrm{H}), 1.1(\mathrm{~s}, 3 \mathrm{H}), 2.2(2 \mathrm{H}, J=16 \mathrm{~Hz}), 3.1(\mathrm{~d}, 1 \mathrm{H}$, $J=16 \mathrm{~Hz}), 3.3(\mathrm{~d}, 1 \mathrm{H}), 3.8(\mathrm{~S}, 3 \mathrm{H}), 6.3(\mathrm{~s}, 1 \mathrm{H}), 6.9(\mathrm{~m}, 2 \mathrm{H})$, $7(\mathrm{~s}, 1 \mathrm{H}), 7.1(\mathrm{~m}, 2 \mathrm{H}), 7.7\left(\mathrm{dd}, 1 \mathrm{H}, J_{1}=12 \mathrm{~Hz}, J_{2}=2 \mathrm{~Hz}\right)$, $7.8(\mathrm{~d}, 1 \mathrm{H}, J=1.6 \mathrm{~Hz}), 7.9(\mathrm{~m}, 2 \mathrm{H}), 8.1(\mathrm{~m}, 1 \mathrm{H}), 8.2(\mathrm{~m}, 1 \mathrm{H})$, $10.4(\mathrm{~s}, 1 \mathrm{H}) ;{ }^{13} \mathrm{C}$ NMR $(\delta$, ppm in DMSO-d 6$): 28.1,28.7$, 34.7, 37.7, 50.6, 56.8, 63.9, 113.4, 117, 118.1, 123.2, 126.3, 127.2, 128, 129.0, 129.5, 129.6, 129.8, 131, 133.7, 134.2, 135, $140,152,154.3,155.9,156.6,192.2$.

5-(2,2-dimethyl-4,7,12-trioxo-1,2,3,4,5,7,12,14-octahydrophthalazino(2,3-b)phthalazin-5-yl)-2-methoxy-N-(4-methoxyphenyl)benzenesulfonamide (6f): M.p.: $244-246^{\circ} \mathrm{C}$; IR $\left(\mathrm{cm}^{-1}\right):\left(v_{\mathrm{N}-\mathrm{H}} 3249\right), v_{\mathrm{C}-\mathrm{H}} 2958, v_{\mathrm{C}=\mathrm{O}} 1665, v_{\mathrm{C}=\mathrm{O}} 1627$, $v_{\mathrm{C}=\mathrm{C}} 1605, v_{\mathrm{SO} 2} 1363,1154 ;{ }^{1} \mathrm{HNMR}(\delta, \mathrm{ppm}$ in DMSO-d 6 ): $1(\mathrm{~s}, 3 \mathrm{H}), 1.1(\mathrm{~s}, 3 \mathrm{H}), 2.3(2 \mathrm{H}, J=16 \mathrm{~Hz}), 3.1(\mathrm{dd}, 1 \mathrm{H}$, $\left.J_{1}=20 \mathrm{~Hz}, J_{2}=2 \mathrm{~Hz}\right), 3.3(\mathrm{~d}, 1 \mathrm{H}), 3.6(\mathrm{~s}, 3 \mathrm{H}), 3.8(\mathrm{~s}, 3 \mathrm{H})$, $6.2(\mathrm{~s}, 1 \mathrm{H}), 6.6(\mathrm{~m}, 2 \mathrm{H}), 6.9(\mathrm{~m}, 2 \mathrm{H}), 7.1(\mathrm{~d}, 1 \mathrm{H}, J=8 \mathrm{~Hz})$, $7.6\left(\mathrm{dd}, 1 \mathrm{H}, J_{1}=2 \mathrm{~Hz}, J_{2}=8 \mathrm{~Hz}\right), 7.7(\mathrm{~d}, 1 \mathrm{H}, J=2.4 \mathrm{~Hz})$, $7.9(\mathrm{~m}, 2 \mathrm{H}), 8.1(\mathrm{~m}, 1 \mathrm{H}), 7.2(\mathrm{~m}, 1 \mathrm{H}), 9.7(\mathrm{~s}, 1 \mathrm{H}) ;{ }^{13} \mathrm{C} \mathrm{NMR}$ 
$\left(\delta\right.$, ppm in DMSO-d $\left._{6}\right): 28.1,28.6,34.7,37.7,50.6,55.5,56.7$, $63.9,114.5,117.2,122,122,126.8,126.9,127.2,128,129$, $129.4,129.8,130.9,131,134.2,134.3,135,151.9,154.2$, $155.89,156.23,192.2$.

5-(2,2-dimethyl-4,7,12-trioxo-1,2,3,4,5,7,12,14-octahydrophthalazino(2,3-b)phthalazin-5-yl)-2-methoxy-N,N-dipropylbenzenesulfonamide (6g): M.p.: $240-242^{\circ} \mathrm{C}$; IR $\left(\mathrm{cm}^{-1}\right)$ : $v_{\mathrm{C}-\mathrm{H}} 2962, v_{\mathrm{C}=\mathrm{O}} 1660, v_{\mathrm{C}=\mathrm{O}} 1623, v_{\mathrm{C}=\mathrm{C}} 1604, v_{\mathrm{SO} 2} 1357$, $1142 ;{ }^{1} \mathrm{H}$ NMR $(\delta$, ppm in DMSO-d 6$): 0.7(\mathrm{t}, 6 \mathrm{H}), 1(\mathrm{~s}, 3 \mathrm{H})$, $1.1(\mathrm{~s}, 3 \mathrm{H}), 1.3$ (sextet, 4H), $2.2(2 \mathrm{H}, J=16 \mathrm{~Hz}), 3(\mathrm{~m}$, $4 \mathrm{H}), 3.1\left(\mathrm{dd}, 2 \mathrm{H}, J_{1}=21.2 \mathrm{~Hz}, J_{2}=2.4 \mathrm{~Hz}\right), 3.3(\mathrm{~d}, 1 \mathrm{H})$, $3.8(\mathrm{~s}, 3 \mathrm{H}), 6.3(\mathrm{~s}, 1 \mathrm{H}), 7.1(d, 1 \mathrm{H}, J=8 \mathrm{~Hz}), 7.6(\mathrm{dd}, 1 \mathrm{H}$, $\left.J_{1}=10.8 \mathrm{~Hz}, J_{2}=2.4 \mathrm{~Hz}\right), 7.8(\mathrm{~d}, 1 \mathrm{H}, J=2.4 \mathrm{~Hz})$, $7.98(\mathrm{~m}, 2 \mathrm{H}), 8.1(\mathrm{~m}, 1 \mathrm{H}), 8.2(\mathrm{~m}, 1 \mathrm{H}) ;{ }^{13} \mathrm{C} \mathrm{NMR}(\delta, \mathrm{ppm}$ in DMSO-d 6 ): 11.3, 21.7, 27.91, 28.8, 34.7, 37.7, 49.4, 50.7, 56.6, 64.1, 113.3, 117.3, 127.1, 128, 128.1, 129.08, 129.5, $129.5,130.2$, 133.7, 134.2, 135, 151.9, 154.2, 155.9, 156.5, 192.2 .

5-(2,2-dimethyl-4,7,12-trioxo-1,2,3,4,5,7,12,14-octahydrophthalazino(2,3-b)phthalazin-5-yl)-2-methyl-N-(p-tolyl)benzenesulfonamide (6h): M.p.: $190-192^{\circ} \mathrm{C}$; IR $\left(\mathrm{cm}^{-1}\right)$ : $v_{\mathrm{N}-\mathrm{H}} \quad 3252, v_{\mathrm{C}-\mathrm{H}} \quad 2959, v_{\mathrm{C}=\mathrm{O}} \quad 1665, v_{\mathrm{C}=\mathrm{O}} \quad 1635, v_{\mathrm{C}=\mathrm{C}}$ $1610, v_{\mathrm{SO} 2} 1361,1152 ;{ }^{1} \mathrm{H}$ NMR $(\delta, \mathrm{ppm}$ in DMSO-d 6 ): $1(\mathrm{~s}, 3 \mathrm{H}), 1.1(\mathrm{~s}, 3 \mathrm{H}), 2.1(\mathrm{~s}, 3 \mathrm{H}), 2.2(2 \mathrm{H}, J=16 \mathrm{~Hz}), 2-3$ $(3 \mathrm{H}), 3.2(2 \mathrm{H}, J=16 \mathrm{~Hz}), 6.3(\mathrm{~s}, 1 \mathrm{H}), 6.8(\mathrm{~s}, 4 \mathrm{H}), 7.3(\mathrm{~d}$, $1 \mathrm{H}, J=8 \mathrm{~Hz}), 7.6(\mathrm{~d}, 1 \mathrm{H}, J=8 \mathrm{~Hz}), 7.8(\mathrm{~s}, 1 \mathrm{H}), 8(\mathrm{~m}$, $2 \mathrm{H}), 8.1(\mathrm{~m}, 1 \mathrm{H}), 8.3(\mathrm{~m}, 1 \mathrm{H}), 10.3(\mathrm{~s}, 1 \mathrm{H}) ;{ }^{13} \mathrm{C} \mathrm{NMR}(\delta$, ppm in DMSO-d 6 ): 19.8, 20.7, 28.3, 28.4, 34.7, 37.7, 50.6, $64,117.1,119,127.2,128.1,129,129.5,129.8,132.5,132.6$, $133,134.3,135.1,135,136,137,138.5,152,154.2,155.8$, 192.2 .

5-(2,2-dimethyl-4,7,12-trioxo-1,2,3,4,5,7,12,14-octahydrophthalazino(2,3-b)phthalazin-5-yl)-N-(2-methoxyphenyl)2-methylbenzenesulfonamide (6i): M.p.: $188-190^{\circ} \mathrm{C}$; IR $\left(\mathrm{cm}^{-1}\right): v_{\mathrm{N}-\mathrm{H}}$ 3306, $v_{\mathrm{C}-\mathrm{H}}$ 2961, $v_{\mathrm{C}=\mathrm{O}} 1664, v_{\mathrm{C}=\mathrm{O}} 1650$, $v_{\mathrm{SO} 2} 1363,1157 ;{ }^{1} \mathrm{H}$ NMR $(\delta, \mathrm{ppm}$ in DMSO-d 6 ): 1 (s, 3H), 1.1, (s, 3H), $2.2(2 \mathrm{H}, J=16 \mathrm{~Hz}), 2.5$ (s, 3H), $3.2(\mathrm{dd}, 1 \mathrm{H}$, $\left.J_{1}=20 \mathrm{~Hz}, J_{2}=2 \mathrm{~Hz}\right), 3.2(\mathrm{~d}, 1 \mathrm{H}, J=20 \mathrm{~Hz}), 3-4$ (3H), $6.2(\mathrm{~s}, 1 \mathrm{H}), 6.6(\mathrm{~m}, 2 \mathrm{H}), 6.7(\mathrm{~m}, 1 \mathrm{H}), 7$ (m, 1H), 7.3 $(\mathrm{d}, 1 \mathrm{H}, J=8 \mathrm{~Hz}), 7.5(\mathrm{~d}, 1 \mathrm{H}, J=4 \mathrm{~Hz}), 7.6(\mathrm{dd}, 1 \mathrm{H}$, $\left.J_{1}=9.6 \mathrm{~Hz}, J_{2}=2 \mathrm{~Hz}\right), 8(\mathrm{~m}, 2 \mathrm{H}), 8.1(\mathrm{~m}, 1 \mathrm{H}), 8.2(\mathrm{~m}, 1 \mathrm{H})$, $9.4(\mathrm{~s}, 1 \mathrm{H}) ;{ }^{13} \mathrm{C}$ NMR $(\delta, \mathrm{ppm}$ in DMSO-d 6$): 20,28.1,28.6$, 34.7, 37.7, 50.6, 55.6, 64, 112, 117.2, 120.5, 125.5, 125.6, $125.6,126.8,127.3,127.5,128,129.1,129.4,132.6,134.3$, $135.1,135.5,137.4,139.2,151.9,152.8,154.2,155.8,192.1$.

5-(2,2-dimethyl-4,7,12-trioxo-1,2,3,4,5,7,12,14-octahydrophthalazino(2,3-b)phthalazin-5-yl)-2-methyl-N-phenylbenzenesulfonamide (6j): M.p.: $207-209^{\circ} \mathrm{C}$; IR $\left(\mathrm{cm}^{-1}\right): v_{\mathrm{N}-\mathrm{H}}$ $3289, v_{\mathrm{C}-\mathrm{H}} 2961, v_{\mathrm{C}=\mathrm{O}} 1659, v_{\mathrm{SO} 2} 1365,1161 ;{ }^{1} \mathrm{H}$ NMR $\left(\delta\right.$, ppm in DMSO-d $\left.\mathrm{d}_{6}\right):=1.1(\mathrm{~s}, 3 \mathrm{H}), 1.1(\mathrm{~s}, 3 \mathrm{H}), 2.2(2 \mathrm{H}$, $J=16 \mathrm{~Hz}), 2.5(\mathrm{~s}, 3 \mathrm{H}), 3.1\left(\mathrm{dd}, 2 \mathrm{H}, J_{1}=20 \mathrm{~Hz}, J_{2}=\right.$ $2 \mathrm{~Hz}), 3.3(\mathrm{~d}, 1 \mathrm{H}, J=20 \mathrm{~Hz}), 6.3(\mathrm{~s}, 1 \mathrm{H}), 6.8(\mathrm{t}, 1 \mathrm{H}), 6.9$ $(\mathrm{m}, 2 \mathrm{H}), 7(\mathrm{~m}, 2 \mathrm{H}), 7.3(\mathrm{~d}, 1 \mathrm{H}, J=8 \mathrm{~Hz}), 7.6(\mathrm{dd}, 1 \mathrm{H}$, $\left.J_{1}=8 \mathrm{~Hz}, J_{2}=2 \mathrm{~Hz}\right), 7.9(\mathrm{~s}, 1 \mathrm{H}), 8(\mathrm{~m}, 2 \mathrm{H}), 8.1(\mathrm{~m}, 1 \mathrm{H})$, $8.3(\mathrm{~m}, 1 \mathrm{H}), 10.4(\mathrm{~s}, 1 \mathrm{H}) ;{ }^{13} \mathrm{C}$ NMR $(\delta, \mathrm{ppm}$ in DMSO-d 6$)$ : $=19.7,28.2$, 28.5, 34.7, 37.7, 50.6, 64, 118.63, 118.6, 123.5, $127.2,128,128.4,129,129.4,129.5,132.5,133.1,134.3$,
$135,136.1,137,138,138.1,138.2,138.3,152,154.2,155.8$, 192.2 .

5-(2,2-dimethyl-4,7,12-trioxo-1,2,3,4,5,7,12,14-octahydrophthalazino(2,3-b)phthalazin-5-yl)-N-(2,4-dimethylphenyl)2-methylbenzenesulfonamide (6k): M.p.: 155-157 ${ }^{\circ} \mathrm{C}$; IR $\left(\mathrm{cm}^{-1}\right): v_{\mathrm{N}-\mathrm{H}} 3250, v_{\mathrm{C}-\mathrm{H}} 2959, v_{\mathrm{C}=\mathrm{O}} 1665, v_{\mathrm{SO} 2} 1361$, $1156 ;{ }^{1} \mathrm{H}$ NMR $(\delta, p p m$ in DMSO-d 6$): 1(\mathrm{~s}, 3 \mathrm{H}), 1.1(\mathrm{~s}, 3 \mathrm{H})$, $1.9(\mathrm{~s}, 3 \mathrm{H}), 2.1(\mathrm{~s}, 3 \mathrm{H}), 2.3(2 \mathrm{H}, J=16 \mathrm{~Hz}), 2.4(\mathrm{~s}, 3 \mathrm{H}), 3.2$ $(2 \mathrm{H}, J=16 \mathrm{~Hz}), 6.3(\mathrm{~s}, 1 \mathrm{H}), 6.6(\mathrm{~m}, 2 \mathrm{H}), 6.8(\mathrm{~s}, 1 \mathrm{H}), 7.3(\mathrm{~d}$, $1 \mathrm{H}, J=12 \mathrm{~Hz}), 7.6(\mathrm{~d}, 2 \mathrm{H}, J=6 \mathrm{~Hz}), 8(\mathrm{~m}, 2 \mathrm{H}), 8.1(\mathrm{~m}, 1 \mathrm{H})$, $8.2(\mathrm{~m}, 1 \mathrm{H}), 9.4(\mathrm{~s}, 1 \mathrm{H}) ;{ }^{13} \mathrm{C} \mathrm{NMR}(\delta, \mathrm{ppm}$ in DMSO-d 6$)$ : 17.9, 20.2, 20.8, 28.1, 28.6, 34.7, 37.7, 50.6, 64.1, 117.2, $126.7,127.1,127.2,127.5,128,129,129.4,131.5,132.4$, $132.6,132.9,134.3,134.4,135.1,135.87,136.19,136.99$, $151.95,154.27,155.86,192.14$.

N-(3-chlorophenyl)-5-(2,2-dimethyl-4,7,12-trioxo-1,2,3, 4,5,7,12,14-octahydrophthalazino(2,3-b)phthalazin-5-yl)-2methylbenzenesulfonamide (6l): M.p.: 225-227 C; IR $\left(\mathrm{cm}^{-1}\right): v_{\mathrm{N}-\mathrm{H}}$ 3269, $v_{\mathrm{C}-\mathrm{H}} 2959, v_{\mathrm{C}=\mathrm{O}} 1665, v_{\mathrm{C}=\mathrm{O}} 1626$, $v \mathrm{C}=\mathrm{C}$ 1596), $\quad \mathrm{SOO}_{2}$ 1361, 1150; ${ }^{1} \mathrm{H} \mathrm{NMR}(\delta, \mathrm{ppm}$ in DMSO-d $\left._{6}\right): 1(\mathrm{~s}, 3 \mathrm{H}), 1(\mathrm{~s}, 3 \mathrm{H}), 2.2(2 \mathrm{H}, J=16 \mathrm{~Hz}), 2.5$ $(\mathrm{s}, 3 \mathrm{H}), 3.1\left(\mathrm{dd}, 1 \mathrm{H}, J_{1}=20 \mathrm{~Hz}, J_{2}=2.4 \mathrm{~Hz}\right), 3.3(\mathrm{~d}, 1 \mathrm{H}$, $J=20 \mathrm{~Hz}), 6.3(\mathrm{~s}, 1 \mathrm{H}), 6.9(\mathrm{~m}, 2 \mathrm{H}), 6.9(\mathrm{~m}, 1 \mathrm{H}), 7(\mathrm{t}, 1 \mathrm{H})$, $3.7(\mathrm{~d}, 1 \mathrm{H}, J=8 \mathrm{~Hz}), 7.6\left(\mathrm{dd}, 1 \mathrm{H}, J_{1}=8 \mathrm{~Hz}, J_{2}=2 \mathrm{~Hz}\right)$, $7.9(\mathrm{~d}, 1 \mathrm{H}, J=1.6 \mathrm{~Hz}), 7.9(\mathrm{~m}, 2 \mathrm{H}), 8(\mathrm{~m}, 1 \mathrm{H}), 8.2(\mathrm{~m}, 1 \mathrm{H})$, $10.7(\mathrm{~s}, 1 \mathrm{H}) ;{ }^{13} \mathrm{C}$ NMR $(\delta, \mathrm{ppm}$ in DMSO-d 6 ): 19.7, 28.2, 28.5, 34.7, 37.7, 50.6, 64, 117.9, 123.3, 127.3, 128, 128.2, $129,129.5,131.2,131,133.2,133.8,134.3,135,136.2,137.1$, $137.1,137.87,139.5,139.6,152.1,154.3,155.9,192.2$.

5-(2,2-dimethyl-4,7,12-trioxo-1,2,3,4,5,7,12,14-octahydrophthalazino(2,3-b)phthalazin-5-yl)-N-(4-methoxyphenyl)2-methylbenzenesulfonamide (6m): M.p.: $180-182^{\circ} \mathrm{C}$; IR $\left(\mathrm{cm}^{-1}\right):\left(v_{\mathrm{N}-\mathrm{H}} 3270, v_{\mathrm{C}-\mathrm{H}} 2958, v_{\mathrm{C}=\mathrm{O}} 1667, v_{\mathrm{SO} 2} 1363\right.$, 1148; ${ }^{1} \mathrm{H}$ NMR $(\delta$, ppm in DMSO-d 6$): 1(\mathrm{~s}, 3 \mathrm{H}), 1.1(\mathrm{~s}, 3 \mathrm{H})$, $2.2(2 \mathrm{H}, J=16 \mathrm{~Hz}), 3.1\left(\mathrm{dd}, 1 \mathrm{H}, J_{1}=20 \mathrm{~Hz}, J_{2}=2 \mathrm{~Hz}\right)$, $3.2(\mathrm{~d}, 1 \mathrm{H}, J=19 \mathrm{~Hz}), 3.3(\mathrm{~s}, 3 \mathrm{H}), 3.6(\mathrm{~s}, 3 \mathrm{H}), 6.3(\mathrm{~s}, 1 \mathrm{H}), 6.6$ $(\mathrm{m}, 2 \mathrm{H}), 6.8(\mathrm{~d}, 2 \mathrm{H}, J=3.2 \mathrm{~Hz}), 7.3(\mathrm{~d}, 1 \mathrm{H}, J=8 \mathrm{~Hz}), 7.6$ $\left(\mathrm{dd}, 1 \mathrm{H}, J_{1}=9.6 \mathrm{~Hz}, J_{2}=1.6 \mathrm{~Hz}\right), 7.8(\mathrm{~d}, 1 \mathrm{H}, J=1.6 \mathrm{~Hz})$, $8(\mathrm{~m}, 2 \mathrm{H}), 8.1(\mathrm{~m}, 1 \mathrm{H}), 8.3(\mathrm{~m}, 1 \mathrm{H}), 10(\mathrm{~s}, 1 \mathrm{H}) ;{ }^{13} \mathrm{C} \mathrm{NMR}(\delta$, ppm in DMSO-d 6 ): 19.9, 28.3, 28.4, 34.7, 37.7, 50.6, 55.5, $64.0,114.6,115,117.1,121.8,127.2,128,129,129.4,130.7$, $132.4,132.9,134.3,135.1,136,137,138.5,152,154.3,155.9$, 156.2, 192.2.

5-(2,2-dimethyl-4,7,12-trioxo-1,2,3,4,5,7,12,14-octahydrophthalazino(2,3-b)phthalazin-5-yl)-2-methyl-N,N-dipropylbenzenesulfonamide (6n): M.p.: $170-172^{\circ} \mathrm{C}$; IR $\left(\mathrm{cm}^{-1}\right)$ : $v_{\mathrm{C}-\mathrm{H}} \quad 2963, v_{\mathrm{C}=\mathrm{O}}$ 1664, $v_{\mathrm{SO} 2} 1363,1144, v_{\mathrm{C}-\mathrm{H}} 3091$, $v \mathrm{C}-\mathrm{H} \quad 2850, v_{\mathrm{C}=\mathrm{O}} 1664, v_{\mathrm{C}=\mathrm{C}} 1601, v_{\mathrm{SO} 2} 1368,1169$; ${ }^{1} \mathrm{H}$ NMR $(\delta, \mathrm{ppm}$ in DMSO-d 6$)$ : $0.6(\mathrm{t}, 6 \mathrm{H}), 1.1(\mathrm{~s}, 3 \mathrm{H})$, 1.1 (s, 3H), 1,3 (sextet, 4H), $2.2(2 \mathrm{H}, J=16 \mathrm{~Hz}), 2.4(\mathrm{~s}$, $3 \mathrm{H}), 3.1(\mathrm{q}, 4 \mathrm{H}), 3.2\left(1 \mathrm{H}, \mathrm{dd}, J_{1}=20 \mathrm{~Hz}, J_{2}=2 \mathrm{~Hz}\right)$, $3.3(\mathrm{~d}, 1 \mathrm{H}), 6.3(\mathrm{~s}, 1 \mathrm{H}), 7.3(\mathrm{~d}, 1 \mathrm{H}, \mathrm{J}=8 \mathrm{~Hz}), 7.6(1 \mathrm{H}, \mathrm{dd}$, $\left.J_{1}=8 \mathrm{~Hz}, J_{2}=1.6 \mathrm{~Hz}\right), 7.8(\mathrm{~d}, 1 \mathrm{H}, J=2 \mathrm{~Hz}), 7.9(\mathrm{~m}, 2 \mathrm{H})$, $8.1(\mathrm{~m}, 1 \mathrm{H}), 8.2(\mathrm{~m}, 1 \mathrm{H}) ;{ }^{13} \mathrm{C} \mathrm{NMR}(\delta, \mathrm{ppm}$ in DMSO-d 6$)$ : $11.2,19.9,21.4,28,28.6,34.7,37.7,48.7,50.6,64.2,117.2$, $127.2,128,128.2,129,129.5,131,5,133.2,134.3,135.1$, $136.1,137,138.4,152,154.3,155.9,192.2$. 


\subsection{Biological evaluation}

2.6a Antibacterial activity: The phthalazine sulfonamides were evaluated for their antibacterial activity against $S$. aureus as Gram-positive bacteria and as Gram-negative bacteria. The antibacterial efficiency of the synthesized compounds is assessed by disc-diffusion method (NCCLS, 1997) using Nutrient agar medium for bacteria. Different concentrations of phthalazine sulfonamides were prepared in sterilized Mueller-Hinton broth at final concentrations of 5, 2.5, 1.25, 0.62 and $0.31 \mathrm{mg} / \mathrm{mL}$. Firstly, the compounds were dissolved in DMSO. Antimicrobial activity was measured at $150 \mu \mathrm{g} /$ disc concentrations. Disc containing DMSO was used as control. To ensure that the solvent had no effect on the bacterial growth, a control test was performed with a test medium supplemented with DMSO at the same dilutions as used in the experiment. The tested bacteria were transferred to tubes containing 4 to $5 \mathrm{~mL}$ Nutrient Broth. The test cultures were incubated at $37^{\circ} \mathrm{C}$ until they were visibly turbid. The density of these cultures were adjusted to $0.5 \mathrm{McF}$ arland with sterile saline. After autoclaving, nutrient was poured into Petri dishes to give a uniform depth of approximately $4 \mathrm{~mm}$ and was allowed to a cool temperature. The bacteria are spread on the surface of agar medium and incubated for $24 \mathrm{~h}$ at $37^{\circ} \mathrm{C}$. After incubation, the growth inhibition zone around the disc was observed indicating that the examined compounds inhibit the growth of microorganisms.

\section{Results and Discussion}

\subsection{Synthesis and characterization of diatomite $-\mathrm{SO}_{3} \mathrm{H}$}

Diatomite, also known as diatomaceous earth, is a fossil assemblage of siliceous diatom frustules and is a kind of natural nanoporous material. A new heterogeneous solid acid catalyst has been prepared from the reaction of diatomite and chlorosulfonic acid (Scheme 2).

The acid sites content of diatomite- $\mathrm{SO}_{3} \mathrm{H}$ was estimated by back titration. To a solution of $\mathrm{NaOH}(0.1 \mathrm{M})$ was added to $0.02 \mathrm{~g}$ of diatomite- $\mathrm{SO}_{3} \mathrm{H}$ and stirred for $15 \mathrm{~min}$. The catalyst was separated, washed with deionized water and the excess of $\mathrm{NaOH}$ was titrated with $\mathrm{HCl}$ in the presence of phenol phthalein as an indicator. The number of $\mathrm{H}^{+}$sites was obtained as $0.2 \mathrm{mmol} / \mathrm{g}$.

The characterization of diatomite- $\mathrm{SO}_{3} \mathrm{H}$ was performed by fourier transform spectroscopy (FT-IR). After

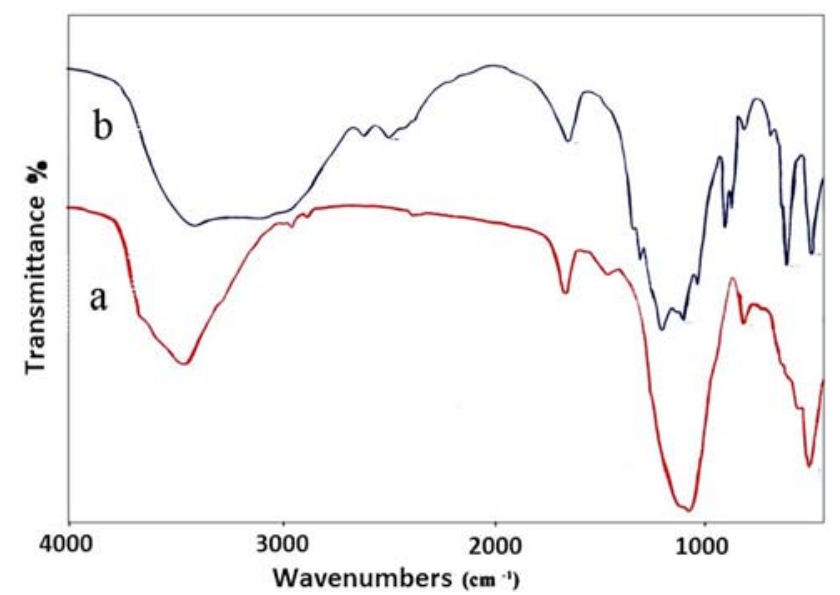

Figure 2. Comparative FT-IR spectra of (a) diatomite and (b) diatomite- $\mathrm{SO}_{3} \mathrm{H}$.

functionalization of diatomite, new bands are clearly seen. The presence of the sulfonic acid group is demonstrated by the bands at 1071,1175 and $579 \mathrm{~cm}^{-1}$, which correspond to the symmetric and asymmetric $\mathrm{SO}_{2}$ and also C-S stretching modes, respectively (Figure 2).

\subsection{Synthesis of $2 H$-indazolo $(2,1-b)$ phthalazine-triones}

Several reaction parameters such as solvent, temperature and catalyst amount were optimized in the reaction of dimedone (1 mmol), 2-bromobenzaldehyde (1 $\mathrm{mmol})$, and phthalhydrazide $(1 \mathrm{mmol})$ in the presence of diatomite- $\mathrm{SO}_{3} \mathrm{H}$ as a model reaction. Firstly, the reaction was studied in the presence of different amount of catalyst. The best result was obtained by using $0.3 \mathrm{~g}$ of diatomite- $\mathrm{SO}_{3} \mathrm{H}$ at $80^{\circ} \mathrm{C}$ (Table 1, entry 5) while a higher amount of catalyst did not affect the product yield.

To find the best solvent, the model reaction was carried out in various solvents. The best result was obtained in ethanol as protic solvent (Table 2, entry 5). Also, the reaction was tested under solvent-free conditions and the product was obtained in very low yield (Table 2, entry 1).

Also, the effects of different temperatures were studied in the model reaction. It was observed that the best

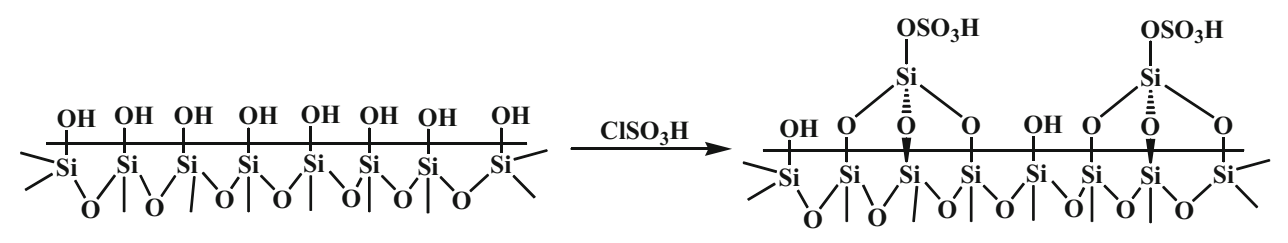

Scheme 2. Preparation of diatomite- $\mathrm{SO}_{3} \mathrm{H}$. 
Table 1. The synthesis of phthalazine using different amounts of catalyst ${ }^{\mathrm{a}}$.

\begin{tabular}{llcc}
\hline Entry & Diatomite-SO $\mathrm{SO}_{3} \mathrm{H}(\mathrm{g})$ & Time $(\mathrm{h})$ & Yield $^{\mathrm{b}}(\%)$ \\
\hline 1 & 0.05 & 6 & 25 \\
2 & 0.1 & 4 & 46 \\
3 & 0.2 & 4 & 68 \\
4 & 0.3 & 4 & 83 \\
5 & 0.3 & 3 & 83 \\
6 & 0.3 & 2.5 & 83 \\
7 & 0.35 & 4 & 83 \\
8 & 0.4 & 4 & 83 \\
9 & 0.5 & 4 & 83 \\
\hline
\end{tabular}

a) Reaction condition:dimedone (1 mmol), 2- bromobenzaldehyde $(1 \mathrm{mmol})$, phthalhydrazide $(1 \mathrm{mmol})$ and $80^{\circ} \mathrm{C}$. b) Isolated yield.

Table 2. The effect of various solvent in the synthesis of phthalazine $^{\mathrm{a}}$.

\begin{tabular}{lcc}
\hline Entry & Solvent & Yield $^{\mathrm{b}}(\%)$ \\
\hline 1 & Solvent-free & 24 \\
2 & $\mathrm{DMF}$ & 32 \\
3 & $\mathrm{CH}_{2} \mathrm{Cl}_{2}$ & 50 \\
4 & $\mathrm{H}_{2} \mathrm{O} / \mathrm{Ethanol}$ & 57 \\
5 & Ethanol & 83 \\
\hline
\end{tabular}

a) Reaction condition: dimedone (1 mmol), 2- bromobenzaldehyde (1 mmol), phthalhydrazide $(1 \mathrm{mmol}), 2.5 \mathrm{~h}$, reflux. b) Isolated yield.

Table 3. The synthesis of phthalazine-trione at different temperatures ${ }^{\mathrm{a}}$.

\begin{tabular}{lcc}
\hline Entry & Temperature $\left({ }^{\circ} \mathrm{C}\right)$ & Yield $^{\mathrm{b}}(\%)$ \\
\hline 1 & 25 & 0 \\
2 & 50 & 40 \\
3 & 60 & 64 \\
4 & reflux & 83 \\
\hline
\end{tabular}

a) Reaction condition: dimedone (1 mmol), 2- bromobenzaldehyde (1 mmol), phthalhydrazide (1 mmol), $2.5 \mathrm{~h}$, ethanol. b) Isolated yield.

result was obtained under reflux conditions (Table 3, entry 4).

After optimization of the reaction conditions, the reaction of dimedone $(1 \mathrm{mmol})$, phthalhydrazide (1 mmol) and different aldehydes was studied. The results were summarized in Table 4.

As can be seen in this Table, the corresponding phthalazine-triones derivatives were synthesized by the one-pot three-component condensation in good to high yields in the presence of diatomite- $\mathrm{SO}_{3} \mathrm{H}$ as solid acid catalyst. The aldehyde with both electron releasing and electron withdrawing substituents on the aryl ring were tolerated and successfully reacted under optimized conditions. Benzaldehydes bearing electron withdrawing substituent (such as $\mathrm{NO}_{2}$ ) and halogen (Table 4, entries; $3,7,8,9,10$ and 12) afforded the product with slightly better yields. The presence of electron-donating substituent (such as $\mathrm{OH}$ ) was decreased the yield of product (Table 4, entry 13).

\subsection{Synthesis of phthalazine sulfonamides}

In the next step of this work, several phthalazine sulfonamides were synthesized. Phthalazines obtained from 4-methoxy and 4-methyl benzaldehyde (Table 4, entries 1 and 2, $4 \mathbf{a}$ and $\mathbf{4 b}$ ), were selected as the main structures and were converted to different sulfonamides in 2 steps. Firstly, these phthalazins were sulfonated with chlorosulfonic acid as sulfonating agent given the corresponding sulfonylchlorides (5a and $\mathbf{5 b}$ ) in high purity and used in the next step without any purification. For the synthesis of the corresponding sulfonamides $(6 \mathbf{6}-$ n) the synthesized sulfonylchloride (5a and5b) were reacted with various amines in the presence of a suitable base at room temperature under solvent-free conditions. ${ }^{26}$ To find the best solid base, p-toluidine was treated with5a in the presence of different amount of $\mathrm{NaHCO}_{3}$ and $\mathrm{K}_{2} \mathrm{CO}_{3}$ and the results were summarized in Table 5. As can be seen in this table when $\mathrm{K}_{2} \mathrm{CO}_{3}$ was used as a base the product was obtained in higher yield after shorter reaction time compared to $\mathrm{NaHCO}_{3}$.

A series of aliphatic and aromatic amines containing electron-withdrawing and electron-donating substituent was also examined to expand the synthetic utility of the protocol (Table 6). As can be seen in this table, aromatic amines are more active than aliphatic amines and the corresponding sulfonamides were obtained in higher yield after shorter reaction time. Also, both electron releasing and electron withdrawing groups were tolerated and successfully reacted with sulfonyl chloride under optimized conditions.

\subsection{Biological evaluation}

The antibacterial activity of the novel phthalazine sulfonamides (6a-n) was evaluated against $S$. aureus and $E$. coli using the agar diffusion method. The diameter of the zone of inhibition considered and $\mathrm{IC}_{50}$ values were calculated and reported in Table S1. Among the 14 tested compounds, $6 \mathrm{~b}$ is found with 23 and $15 \mathrm{~mm}$ diameter for $S$. aureus and E.coli, respectively which show the highest diameter of zone of inhibition where 
Table 4. Synthesis of $2 \mathrm{H}$-indazolo (2,1-b) phthalazine-triones $(\mathbf{4 a - 4 o})$ in the presence of diatomite- $\mathrm{SO}_{3} \mathrm{H}$ at $80^{\circ} \mathrm{C}$.

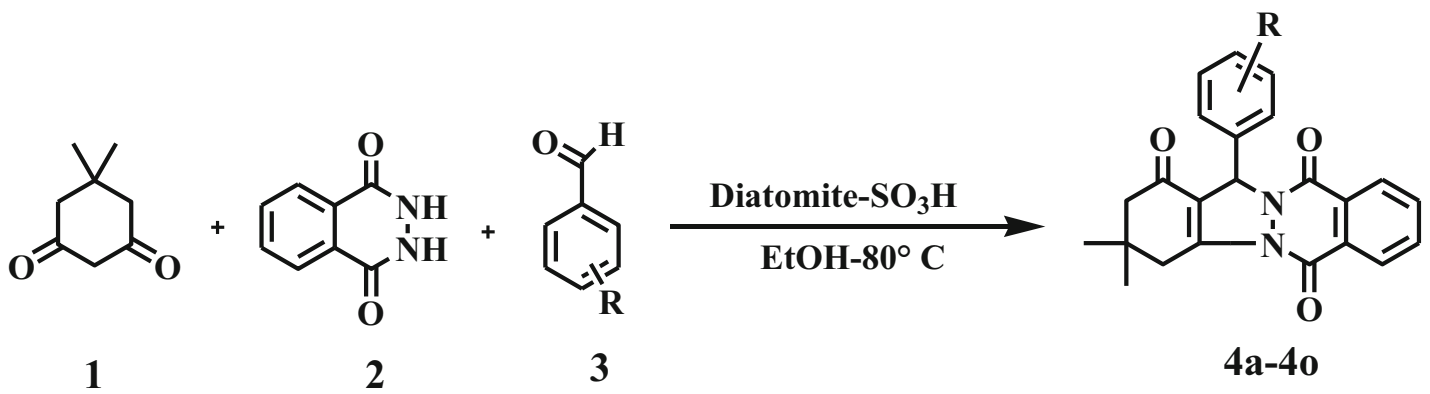

$\mathrm{R}=\mathrm{OMe}, \mathrm{Me}, \mathrm{Br}, \mathrm{Cl}, \mathrm{NO}_{2}, \mathrm{CN}, \mathrm{OH}$

\begin{tabular}{lcccccc}
\hline Entry & $\mathrm{R}$ & Product & Time $(\mathrm{h})$ & Yield $^{\mathrm{a}}(\%)$ & M.p. $\left({ }^{\circ} \mathrm{C}\right)$ & M.p. $\left({ }^{\circ} \mathrm{C}\right)(\mathrm{Lit})$ \\
\hline 1 & $4-\mathrm{MeO}$ & $\mathbf{4 a}$ & 3 & 78 & $220-222$ & $220-222^{26}$ \\
2 & $4-\mathrm{Me}$ & $\mathbf{4 b}$ & 3 & 77 & $228-230$ & $226-228^{27}$ \\
3 & $4-\mathrm{Br}$ & $\mathbf{4 c}$ & 2.5 & 83 & $262-264$ & $258-260^{27}$ \\
4 & $4-\mathrm{Cl}$ & $\mathbf{4 d}$ & 2.5 & 81 & $260-262$ & $264-266^{27}$ \\
5 & $3,4,5-(\mathrm{MeO})_{3}$ & $\mathbf{4 e}$ & 3 & 75 & $232-234$ & $232-234^{27}$ \\
6 & $\mathrm{H}$ & $\mathbf{4 f}$ & 3 & 79 & $205-207$ & $207-209^{27}$ \\
7 & $3-\mathrm{NO}_{2}$ & $\mathbf{4 g}$ & 2.5 & 80 & $268-270$ & $270-272^{27}$ \\
8 & $2-\mathrm{NO}_{2}$ & $\mathbf{4 h}$ & 2.5 & 81 & $245-247$ & $251-253^{27}$ \\
9 & $4-\mathrm{NO}_{2}$ & $\mathbf{4 i}$ & 2.5 & 82 & $220-222$ & $217-219^{27}$ \\
10 & $2,-(\mathrm{Cl})_{2}$ & $\mathbf{4 j}$ & 2.5 & 82 & $215-217$ & $219-221^{27}$ \\
11 & $2-\mathrm{Cl}$ & $\mathbf{4 k}$ & 2.5 & 80 & $262-264$ & $264-266^{27}$ \\
12 & $2,6-(\mathrm{Cl})_{2}$ & $\mathbf{4 l}$ & 2.5 & 82 & $263-265$ & $260-262^{28}$ \\
13 & $4-\mathrm{OH}$ & $\mathbf{4 m}$ & 3 & 73 & $261-263$ & $261-263^{29}$ \\
14 & $4-\mathrm{CN}$ & $\mathbf{4 n}$ & 2.5 & 81 & $228-230$ & $224-226^{28}$ \\
15 & $3-(\mathrm{MeO})_{2}$ & $\mathbf{4 o}$ & 3 & 78 & $236-238$ & $234^{30}$ \\
\hline
\end{tabular}

a) Reaction condition: dimedone ( $1 \mathrm{mmol})$, aromatic aldehyde $(1 \mathrm{mmol})$, phthalhydrazide $(1 \mathrm{mmol})$, ethanol, $80^{\circ} \mathrm{C}$.

b) Isolated yield.

Table 5. Reaction of p-toluidine with $\mathbf{5 a}$ in the presence of $\mathrm{NaHCO}_{3}$ and $\mathrm{K}_{2} \mathrm{CO}_{3}$ under solvent-free conditions.

\begin{tabular}{cccccc}
\hline Entry & $\mathrm{NaHCO}_{3} / \mathrm{K}_{2} \mathrm{CO}_{3}(\mathrm{mmol})$ & Time (min) $\mathrm{NaHCO}_{3}$ & Time (min) $\mathrm{K}_{2} \mathrm{CO}_{3}$ & Yield $^{\text {a }}(\%) \mathrm{NaHCO}_{3}$ & Yield $^{\text {a }}(\%) \mathrm{K}_{2} \mathrm{CO}_{3}$ \\
\hline 1 & 1 & 60 & 45 & 34 & 60 \\
2 & 2 & 50 & 35 & 53 & 80 \\
3 & 3 & 25 & 25 & 76 & 93 \\
4 & 4 & 25 & 25 & 80 & 93
\end{tabular}

a) Reaction condition: p-Toluidine $(1 \mathrm{mmol})$, 5-(2,2-dimethyl-4,7,12-trioxo-1,2,3,4,5,7,12,14-octahydrophthalazino(2,3b)phthalazin-5-yl)-2-methoxybenzene-1-sulfonyl chloride (1 mmol), solvent-free, room temperature. b) Isolated yield.

the corresponding $\mathrm{IC}_{50}$ 's are calculated as 10.2 and 4.2 $\mu \mathrm{g} / \mathrm{mL}$ which are considerably low, as can be seen in Table S1. The same sample at different concentration was measured repeatedly for $\mathrm{IC}_{50}$ values. The concentration of tested samples $(\mu \mathrm{g} / \mathrm{mL})$ giving $50 \%$ inhibition were calculated from the quadratic curve fitting regression line with more of logarithmic concentration plotted versus inhibition percentage. The confidence bound of the quadratic curve fitting is more than $95 \%$ and root mean square error (rmse) is 5.32 which shows a trustful curve fitting. The concentration of $\mathrm{IC}_{50}$ is found to be $0.3503 \mu \mathrm{g} / \mathrm{mL}$.

The in vitro efficiency of these novel phthalazine sulfonamides against the Gram-negative bacteria was much lower than against Gram-positive bacteria. Compounds containing methyl and methoxy group $(\mathbf{6 a}, \mathbf{6 b}, \mathbf{6 f})$ showed higher activity against gram-positive bacteria. Also, these results show that phthalazine sulfonamides derived from aliphatic amines are less active than those from aromatic amine $(\mathbf{6 g}, \mathbf{6 n})$. 
Table 6. Synthesis of phthalazine sulfonamides $(\mathbf{6 a}-\mathbf{n})$ at room temperature under solvent-free conditions.<smiles>[R]c1ccc(C2C3=C(CC(C)(C)CC3=O)n3c(=O)c4ccccc4c(=O)n32)cc1</smiles>

$4 \mathrm{a}, 4 \mathrm{~b}$

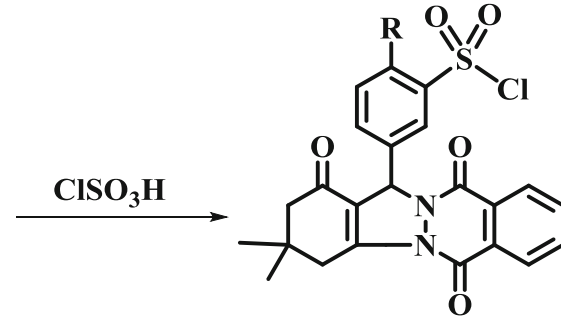

$\mathbf{5 a}, \mathbf{5 b}$

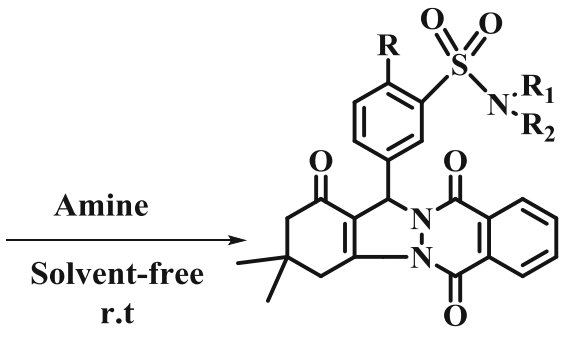

6a-6n

$\mathrm{R}_{1}=\mathrm{H}, \mathrm{CH}_{3} \mathrm{CH}_{2} \mathrm{CH}_{2}$ $\mathrm{R}_{2}=\mathrm{H}, \mathrm{Ph}\left(\mathrm{CH}_{3}\right), \operatorname{Ph}\left(\mathrm{OCH}_{3}\right), \operatorname{Ph}(\mathrm{Cl}), \operatorname{Ph}\left(\mathrm{CH}_{3}\right)_{2}$, $\mathrm{CH}_{3} \mathrm{CH}_{2} \mathrm{CH}_{2}$

\begin{tabular}{lcccc}
\hline Entry & Amines & Product & Time $(\mathrm{min})$ & Yield $^{\text {a }}(\%)$ \\
\hline 1 & p-toluidine & $\mathbf{6 a}$ & 25 & 93 \\
2 & o-anisidine & $\mathbf{6 b}$ & 20 & 96 \\
3 & Aniline & $\mathbf{6 c}$ & 25 & 92 \\
4 & 2,4-dimethyl aniline & $\mathbf{6 d}$ & 25 & 92 \\
5 & 3-chloro aniline & $\mathbf{6 e}$ & 40 & 83 \\
6 & p-anisidine & $\mathbf{6 f}$ & 20 & 92 \\
7 & Dipropylamine & $\mathbf{6 g}$ & 25 & 72 \\
8 & p-toluidine & $\mathbf{6 h}$ & 25 & 90 \\
9 & o-anisidine & $\mathbf{6 i}$ & 20 & 94 \\
10 & Aniline & $\mathbf{6 j}$ & 20 & 90 \\
11 & 2,4-dimethyl aniline & $\mathbf{6 k}$ & 20 & 86 \\
12 & 3-chloro aniline & $\mathbf{6}$ & 30 & 94 \\
13 & o-anisidine & $\mathbf{6 m}$ & 20 & 76 \\
14 & Dipropylamine & $\mathbf{6 n}$ & 35 & 95 \\
\hline
\end{tabular}

a) Reaction condition: phthalazinsulfonyl chloride ( $1 \mathrm{mmol})$, various amines $(1 \mathrm{mmol}), \mathrm{K}_{2} \mathrm{CO}_{3}(3 \mathrm{mmol})$, solvent-free, room temperature.

b) Isolated yield.

\subsection{Statistical analysis}

The effect of concentration on inhibition zone in both Gram positive and Gram negative bacteria was shown in (Figure S4). Bar chart of the effects of various compounds on inhibition zone in both Gram positive and Gram negative bacteria was shown in (Figure S5). According to this figure, Gram-positive bacteria are more potent than Gram-negative bacteria and $\mathbf{6 f}$ was the best antibacterial compound in our in vitro investigation. After the evaluation given above, statistical analysis of antibacterial effect was performed and is shown in Table S2.

\subsection{Physicochemical properties}

In silico physicochemical parameters of synthesized compounds were performed to predict absorption and permeability. In this study, the logarithm of partition coefficient (milogP), molecular weight (MW), number of hydrogen bond acceptors (n-ON), number of hydrogen bond donors (n-OHNH), number of rotatable bonds (n-rotb), topological polar surface area (TPSA), and Lipinski's rule of five 22 were calculated using Molinspiration online property calculation server (http://www. molinspiration.com) and the value obtained is shown in Table S3. A compound likely to be selected as an orally active drug candidate that should show no more than one violation of the following five criteria: $\log$ $\mathrm{Po} / \mathrm{w} \leq 5, \mathrm{MW} \leq 500, \mathrm{n}-$ rotb $\leq 10, \mathrm{n}-\mathrm{ON} \leq 10$ and $\mathrm{n}-\mathrm{OHNH} \leq 5$.

Lipophilicity (hydrophobicity) is an important physicochemical parameter to predict specific behavior of a compound in passive diffusion through the intestinal membrane. It is normally quantified as $\log \mathrm{P}_{\mathrm{o} / \mathrm{w}}$, where $\mathrm{P}$ is the ratio of the concentrations of a compound in a mixture of octanol and water phases at equilibrium. All the synthetic compounds in this study except $5 \mathrm{~K}$ showed suitable lipophilicity for intestinal absorption. Polar surface area (PSA) value is an important property 
for the prediction of oral bioavailability of drug compounds. The polar surface area (PSA), expressed here as topological surface area (TPSA) is obtained from the surface areas that are occupied by oxygen and nitrogen atoms, and by hydrogen atoms connected to them. TPSA values of molecules with less than $140 \AA^{2}$ are recognized to have suitable intestinal absorption, and TPSA less than $60 \AA^{2}$ represent good blood brain barrier penetration. All presented compounds are expected to have good intestinal absorption and have weak blood-brain barrier penetration. Hydrogen bonding capacity of the drug molecules is also a critical factor for drug absorption. The number of hydrogen bond donors is found to be less than 5 and the hydrogen bond acceptors is less than 10. The number of hydrogen bond acceptors of all studied sulfonamides are not more than 10 bonds and also the number of hydrogen bond donors are less than five bonds, and may thus have good intestinal absorption. The number of rotatable bonds is found to be important predictors for the efficient binding to receptors and channels as well as for good oral bioavailability. ${ }^{31}$ Drugs with less than 10 rotatable bonds tend to show good oral bioavailability. All sulfonamide derivatives have good rotatable bonds for oral bioavailability.

Summarizing the physico-chemical properties of studied compounds suggested that all of the compounds obey the Lipinski "rules of five" and have only one violation of the rule and meet all criteria for good permeability, except 6k showing two violations of the above criteria.

\section{Conclusions}

In the present work we have demonstrated an efficient protocol for the synthesis of phthalazines using diatomite- $\mathrm{SO}_{3} \mathrm{H}$. Some features of this method are easy synthetic procedure, high catalyst stability and durability, easy products separation with high purity, and needing only room temperature. Also, several novel phthalazine sulfonamides as biologically important heterocycles were synthesized at room temperature under solvent-free conditions. All the products were obtained in high yield and purity after an easy work-up and screened for their in vitro antibacterial activity against $S$. aureus and E. coli. The results showed that the best activities were related to the phthalazine sulfonamides containing electron-donating groups (OMe and $\mathrm{Me}$ ). A theoretical study was able to check and predict the physico-chemical profile of synthetic derivatives. A careful study suggested that all compounds, except $6 \mathrm{k}$, have only one violation of the rule of five and meet all criteria for good permeability.

\section{Supplementary Information (SI)}

Full set of characterization data (IR, ${ }^{1} \mathrm{H}$ and ${ }^{13} \mathrm{C} N M R$ spectra) are given in electronic Supporting Information available at www.ias.ac.in/chemsci.

\section{Acknowledgements}

We appreciate the financial support of Shahreza Branch, Islamic Azad University (IAUSH) Research Council.

\section{References}

1. Li J, Lou J, Wang Z, Wang T, Xiao Y, Hu X, Liu P and Hong X 2015 Design, synthesis and pharmacological evaluation of novel $\mathrm{N}$-(2-(1,1-dimethyl-5,7-dioxo-4,6diazaspiro [2.4] heptan-6-yl) ethyl) sulfonamide derivatives as potential anticonvulsant agents Eur. J. Med. Chem. 92370

2. Ganguly A K, Alluri S S, Wang C-H, Antropow A, White A, Caroccia D, Biswas D, Kang E, Zhang L-K and Carroll S S 2014 Structural optimization of cyclic sulfonamide based novel HIV-1 protease inhibitors to picomolar affinities guided by X-ray crystallographic analysis Tetrahedron $\mathbf{7 0} 2894$

3. Reddy N D, Shoja M, Biswas S, Nayak P G, Kumar N and Rao C M 2016 An appraisal of cinnamyl sulfonamide hydroxamate derivatives (HDAC inhibitors) for anti-cancer, anti-angiogenic and anti-metastatic activities in human cancer cells Chem. Bio. Interact. 253112

4. Zoumpoulakis P, Camoutsis C, Pairas G, Soković M, Glamočlija J, Potamitis C and Pitsas A 2012 Synthesis of novel sulfonamide-1, 2, 4-triazoles, 1, 3, 4-thiadiazoles and 1,3,4-oxadiazoles, as potential antibacterial and antifungal agents. Biological evaluation and conformational analysis studies Bioorg. Med. Chem. 201569

5. Ning X, Guo Y, Ma X, Zhu R, Tian C, Zhang Z, Wang X, Ma Z and Liu J 2013 Design, synthesis and pharmacological evaluation of (E)-3, 4-dihydroxy styryl sulfonamides derivatives as multifunctional neuroprotective agents against oxidative and inflammatory injury Bioorg. Med. Chem. 215589

6. Yoshino H, Ueda N, Niijima J, Sugumi H, Kotake Y, Koyanagi N, Yoshimatsu K, Asada M and Watanabe T 1992 Novel sulfonamides as potential, systemically active antitumor agents J. Med. Chem. 352496

7. Pandya R, Murashima T, Tedeschi L and Barrett A G 2003 Facile one-pot synthesis of aromatic and heteroaromatic sulfonamides $\mathrm{J}$. Org. Chem. $\mathbf{6 8} 8274$

8. Tang X, Huang L, Qi C, Wu X, Wu W and Jiang H 2013 Copper-catalyzed sulfonamides formation from sodium sulfinates and amines Chem. Comm. 496102

9. Sayyafi M, Seyyedhamzeh M, Khavasi H R and Bazgir A 2008 One-pot, three-component route to $2 \mathrm{H}$-indazolo [2, 1-b] phthalazine-triones Tetrahedron 642375

10. (a) AAl'-Assar F, Zelenin K, Lesiovskaya E, Bezhan I and Chakchir B 2002 Synthesis and pharmacological activity of 1-hydroxy-, 1-amino-, and 1hydrazino-substituted 2, 3-dihydro-1H-pyrazolo [1, 2-a] pyridazine-5, 8-diones and 2, 3-dihydro-1H-pyrazolo [1, 2-b] phthalazine-5, 10-diones Pharm. Chem. J. 36 598; 
(b) Jain R P and Vederas J C 2004 Bioorg. Med. Chem. Lett. 143655

11. Ibrahim H S, Eldehna W M, Abdel-Aziz H A, Elaasser M M and Abdel-Aziz M M 2014 Improvement of antibacterial activity of some sulfa drugs through linkage to certain phthalazin-1 (2H)-one scaffolds Eur. J. Med. Chem. 85 480

12. Xue D-Q, Zhang X-Y, Wang C-J, Ma L-Y, Zhu N, He P, Shao K-P, Chen P-J, Gu Y-F and Zhang X-S 2014 Synthesis and anticancer activities of novel 1, 2, 4-triazolo [3, 4-a] phthalazine derivatives Eur. J. Med. Chem. 85 235

13. Ryu C-K, Park R-E, Ma M-Y and Nho J-H 2007 Synthesis and antifungal activity of 6-arylamino-phthalazine5,8-diones and 6,7-bis (arylthio)-phthalazine-5,8-diones Bioorg. Med. Chem. Lett. 172577

14. Eldehna W M, Ibrahim H S, Abdel-Aziz H A, Farrag N N and Youssef M M 2015 Design, synthesis and in vitro antitumor activity of novel N-substituted-4phenyl/benzylphthalazin-1-ones Eur. J. Med. Chem. 89 549

15. El-Shamy I E, Abdel-Mohsen A, Alsheikh A A, Fouda M M, Al-Deyab S S, El-Hashash M A and Jancar J 2015 Synthesis, biological, anti-inflammatory activities and quantum chemical calculation of some [4-(2, 4, 6trimethylphenyl)-1 (2H)-oxo-phthalazin-2yl] acetic acid hydrazide derivatives Dyes Pigm. 113357

16. Shaterian H R, Ghashang M and Feyzi M 2008 Silica sulfuric acid as an efficient catalyst for the preparation of 2H-indazolo [2, 1-b] phthalazine-triones Appl. Catal., A 345128

17. Ghorbani-Vaghei R, Karimi-Nami R, ToghraeiSemiromi Z, Amiri M and Ghavidel M 2011 One-pot synthesis of aliphatic and aromatic $2 \mathrm{H}$-indazolo [2, 1-b] phthalazine-triones catalyzed by $\mathrm{N}$-halosulfonamides under solvent-free conditions Tetrahedron 671930

18. Reddy M V, Reddy G C S and Jeong Y T 2012 Microwave-assisted, montmorillonite K-10 catalyzed three-component synthesis of $2 \mathrm{H}$-indazolo [2, 1-b] phthalazine-triones under solvent-free conditions Tetrahedron 686820

19. Shekouhy M and Hasaninejad A 2012 Ultrasoundpromoted catalyst-free one-pot four component synthesis of $2 \mathrm{H}$-indazolo [2, 1-b] phthalazine-triones in neutral ionic liquid 1-butyl-3-methylimidazolium bromide Ultrason. Sonochem. 19307

20. Kiasat A R, Noorizadeh S, Ghahremani M and Saghanejad S J 2013 Experimental and theoretical study on one-pot, three-component route to $2 \mathrm{H}$-indazolo [2, 1-b] phthalazine-triones catalyzed by nano-alumina sulforic acid J. Mol. Struct. 1036216

21. Shaterian H R and Aghakhanizadeh M 2012 Four-component synthesis of 2H-indazolo [2, 1-b] phthalazine-1, 6, 11 (13H)-trione derivatives Compt. Rend. Chim. 151060

22. Khurana J M and Magoo D 2009 Efficient one-pot syntheses of $2 \mathrm{H}$-indazolo [2, 1-b] phthalazine-triones by catalytic $\mathrm{H}_{2} \mathrm{SO}_{4}$ in water-ethanol or ionic liquid Tetrahedron Lett. $\mathbf{5 0} 7300$

23. (a) Massah A R, Adibi H, Khodarahmi R, Abiri R, Majnooni M B, Shahidi S, Asadi B, Mehrabi M and Zolfigol M A 2008 Synthesis, in vitro antibacterial and carbonic anhydrase II inhibitory activities of $\mathrm{N}$ acylsulfonamides using silica sulfuric acid as an efficient catalyst under both solvent-free and heterogeneous conditions Bioorg. Med. Chem. 16 5465; (b) Massah A, Dabagh M, Shahidi S, Naghash H J, Momeni A and Aliyan $\mathrm{H} 2009 \mathrm{P}_{2} \mathrm{O}_{5} / \mathrm{SiO}_{2}$ as an efficient and recyclable catalyst for $\mathrm{N}$-acylation of sulfonamides under heterogeneous and solvent-free condition J. Iran. Chem. Soc. 6 405; (c) Maghsoodi N K, Khazaeli T and Massah A R 2015 J. Chem. Res. 39 141; Malekpoor M, Gharaghani S, Sharifzadeh A, Mirsattari S N and Massah A R 2015 Solvent-free synthesis of novel styrenesulfonamide derivatives and evaluation of their antibacterial activit J. Chem. Res. 39433; (d) Massah A R, Gharaghani S, Lordejani H A and Asakere N 2016 New and mild method for the synthesis of alprazolam and diazepam and computational study of their binding mode to GABAA receptor Med. Chem. Res. 251538

24. Ma L-X, Cui B-R, Wu Y, Liu J-C, Cui X, Liu L-P and Piao H-R 2014 Synthesis and positive inotropic evaluation of $[1,2,4]$ triazolo $[3,4-a]$ phthalazine and tetrazolo $[5,1-$ a] phthalazine derivatives bearing substituted piperazine moieties Bioorg. Med. Chem. Lett. 241737

25. Naeimi H and Nazifi Z S 2014 Sulfonated diatomite as heterogeneous acidic nanoporous catalyst for synthesis of 14-aryl-14-H-dibenzo [a, j] xanthenes under green conditions Appl. Catal., A 477132

26. Saha M, Phukan S, Jamatia R, Mitra S and Pal A K 2013 Solvent free, Ni-nanoparticle catalyzed greener synthesis and photophysical studies of novel $2 \mathrm{H}$-indazolo [2, 1-b] phthalazine-trione derivatives $R S C A d v .31714$

27. Shaterian H R, Hosseinian A and Ghashang M 2009 Reusable silica supported poly phosphoric acid catalyzed three-component synthesis of $2 \mathrm{H}$-indazolo [2, 1-b] phthalazine-trione derivatives Arkivoc 259

28. Hasaninejed A, Kazerooni M R and Zare A 2012 Solvent-free, one-pot, four-component synthesis of $2 \mathrm{H}$ indazolo $[2,1-b]$ phthalazine-triones using sulfuric acidmodified PEG-6000 as a green recyclable and biodegradable polymeric catalyst Catal. Today 196148

29. (a) Ziarani G M, Badiei A, Mohtasham N H and Lashgari N 2014 Sulfonic acid functionalized nanoporous silica (SBA-PR-SO3H) as an efficient catalyst for the one-pot synthesis of $2 \mathrm{H}$-indazolo [1, 2-B] phthalazine-triones $J$. Chil. Chem. Soc. 592271

30. (b) Safaei S, Mohammadpoor-Baltork I, Khosropour A R, Moghadam M, Tangestaninejad S and Mirkhani V 2013 Catal. Sci. Technol. 32717

31. Veber D F, Johnson S R, Cheng H-Y, Smith B R, Ward K W and Kopple K D 2002 Molecular properties that influence the oral bioavailability of drug candidates $J$. Med. Chem. 452615 